\title{
ON THE RIEMANN ZETA FUNCTION *
}

BY C. F. CRAIG

In this note a functional relation for the Riemann zeta function is established, and its relation to the well known formula given by Riemann is pointed out. It is believed that the relation is new.

Recall that the even elliptic theta constants are

$$
\left\{\begin{array}{l}
\vartheta_{00}(x)=1+2 \sum_{n=1}^{\infty} q^{4 n^{2}}=1+2 \phi_{00}(x) \\
\vartheta_{01}(x)=2 \sum_{n=1}^{\infty} q^{(2 n-1)^{2}}=2 \phi_{01}(x) \\
\vartheta_{10}(x)=1-2 \sum_{n=1}^{\infty}(-1)^{n-1} q^{4 n^{2}}=1-2 \phi_{10}(x),
\end{array}\right.
$$

with $q=e^{-(\pi / 4) x^{2}}$. These series converge uniformly and absolutely for $0<\delta \leqq x$. Furthermore

$$
\vartheta_{00}\left(\frac{1}{x}\right)=x \vartheta_{00}(x), \quad \vartheta_{01}\left(\frac{1}{x}\right)=x \vartheta_{10}(x), \quad \vartheta_{10}\left(\frac{1}{x}\right)=x \vartheta_{01}(x) .
$$

Let

$$
\vartheta(x)=x^{1 / 2}\left[\vartheta_{01}(x)+\vartheta_{10}(x)-\vartheta_{00}(x)\right] ;
$$

then

$$
\vartheta\left(\frac{1}{x}\right)=\vartheta(x)
$$

Also

$$
1+2 \phi_{00}\left(\frac{1}{x}\right)=x\left[1+2 \phi_{00}(x)\right]
$$

with two similar relations for $\phi_{01}$ and $\phi_{10}$. From these follow

$$
\begin{cases}\lim _{x \rightarrow 0} x \phi_{00}(x)=\frac{1}{2}, & \lim _{x \rightarrow 0} x \phi_{10}(x)=0, \\ \lim _{x \rightarrow 0} x \phi_{01}(x)=\frac{1}{2}, & \lim _{x \rightarrow 0} x^{h} \vartheta(x)=0,\end{cases}
$$

where $h$ is any constant. To prove the last of the expressions

* Presented to the Society, December 27, 1922.

23 
(4) observe that

$$
\vartheta(y)=2 y^{1 / 2} \sum_{1}^{\infty} Q_{n}(y)
$$

where $Q_{n}(y)=q^{(2 n-1)^{2}}-2 q^{4(2 n-1)^{2}}$. For $y \geqq 1, Q_{n}(y)>0$, and hence by (3) $\vartheta(y)$ is never negative. Also

and hence

$$
Q_{n}(y)<e^{-(\pi / 4) y^{2}} e^{-n \pi}, \quad y \geqq 1,
$$

$$
\vartheta(y)<2 y^{1 / 2} e^{-(\pi / 4) y^{2}}\left(1-e^{-\pi / 4}\right)^{-1}, \quad y \geqq 1 .
$$

In $x^{h} \vartheta(x)$ placing $x=1 / y \leqq 1$, and using (3) together with the last inequality, we find

$$
\left|x^{h} \vartheta(x)\right|<2\left(1-e^{-\pi / 4}\right)^{-1}\left|y^{1-h}\right| e^{-(\pi / 4) y^{2}} .
$$

The last of the expressions (4) follows for $y \rightarrow \infty$.

Recall further that

$$
\left\{\begin{aligned}
\zeta(s) & =1+\frac{1}{2^{s}}+\frac{1}{3^{s}}+\frac{1}{4^{s}}+\cdots \\
\left(1-2^{1-s}\right) \zeta(s) & =1-\frac{1}{2^{s}}+\frac{1}{3^{s}}-\frac{1}{4^{s}}+\cdots \\
\left(1-2^{-s}\right) \zeta(s) & =1+\frac{1}{3^{s}}+\cdots
\end{aligned}\right.
$$

and that each of these series converges absolutely and uniformly for $R(s) \geqq 1+\delta, R(s)$ being the real part of $s$. In

$$
\Gamma\left(\frac{s}{2}\right)=\int_{0}^{\infty} e^{-z} z^{(s / 2)-1} d z, \quad R(s)>0,
$$

replace $z$ by $k^{2} \pi x^{2}$ and there results

(6) $\quad \pi^{-s / 2} \Gamma\left(\frac{s}{2}\right) k^{-s}=2 \int_{0}^{\infty} e^{-l l^{2} \pi x^{2}} x^{s-1} d x, \quad R(s)>0$.

In (6) put $k=n$, multiply each side of the equality by $(-1)^{n-1}$, and sum with respect to $n$. This gives, on account of (5),

$$
\left(1-2^{1-s}\right) \chi(s)=\sum_{n=1}^{\infty} 2 \int_{0}^{\infty}(-1)^{n-1} e^{-n^{2} \pi x^{2}} x^{s-1} d x, \quad R(s)>1,
$$


where

$$
\chi(s)=\pi^{-8 / 2} \Gamma\left(\frac{s}{2}\right) \zeta(s) .
$$

An easy investigation * shows that the operations of integration and summation are reversible, giving

$$
\left(1-2^{1-s}\right) \chi(s)=2 \int_{0}^{\infty} \phi_{10}(x) x^{s-1} d x, \quad R(s) \geqq \delta+2 .
$$

The use of (6) with $k=n$ and $2 k=2 n-1$ gives, on summing with respect to $n$,

$$
\begin{aligned}
\chi(s) & =2 \int_{0}^{\infty} \phi_{00}(x) x^{s-1} d x, & & R(s) \geqq \delta+2, \\
2^{s}\left(1-2^{-s}\right) \chi(s) & =2 \int_{0}^{\infty} \phi_{01}(x) x^{s-1} d x, & & R(s) \geqq \delta+2 .
\end{aligned}
$$

The first of this pair of relations is Riemann's well known formula. These three equalities combine to yield, by using the relations (1) and (2),

$$
-\left(2^{s}-1\right)\left(2^{1-s}-1\right) \chi(s)=\int_{0}^{\infty} \vartheta(x) x^{s-\frac{1}{2}} x^{-1} d x .
$$

The derivation of formula (7) is the principal object of this note. Thanks to the last of the relations (4), the integral in (7) converges for every value of $s$ and hence the representation is valid everywhere. In (7) replace $x$ with $1 / x$, use (3) and obtain

$$
-\left(2^{s}-1\right)\left(2^{1-s}-1\right) \chi(s)=\int_{0}^{\infty} \vartheta(x) x^{-\left(s-\frac{1}{2}\right)} x^{-1} d x .
$$

The addition of this equation to (7) gives

$$
\text { (8) }-2\left(2^{s}-1\right)\left(2^{1-s}-1\right) \chi(s)=\int_{0}^{\infty} \vartheta(x)\left[x^{s-\frac{1}{2}}+x^{-\left(s-\frac{1}{2}\right)}\right] x^{-1} d x \text {. }
$$

In this replace $s$ by $1-s$ and we deduce the well known property of $\chi(s)$, viz., $\chi(s)=\chi(1-s)$. Since $\vartheta(x)$ is never

* That given by Whittaker and Watson, Modern Analysis, 3d ed., p. 273 , is readily adapted. 
negative, the right hand member of (7) does not vanish for $s$ real. Thus $\chi(s)$ has a simple pole at $s=0$ and at $s=1$ and has no real zeros. Recalling the location of the poles, all simple, of $\Gamma(s / 2)$, we observe that $\zeta(s)$ has $-2,-4$, $-6, \cdots$ as its only real zeros and that these are simple.

In (8), let $s=\frac{1}{2}+i t$, and we have

$$
X(t)=\int_{0}^{\infty} \vartheta(x) \cos (t \log x) x^{-1} d x,
$$

with

$$
X(t)=\left(2^{1+i t}-1\right)\left(1-2^{\frac{1}{-1}-i t}\right) \chi\left(\frac{1}{2}+i t\right) .
$$

In (9), separate the integral into the two parts from 0 to 1 and from 1 to $\infty$, in the first replace $x$ with $1 / x$, employ (3), and we have

$$
X(t)=2 \int_{1}^{\infty} \vartheta(x) \cos (t \log x) x^{-1} d x .
$$

Now replace $x$ by $e^{x}$, and we obtain

$$
X(t)=2 \int_{0}^{\infty} \vartheta\left(e^{x}\right) \cos (t x) d x .
$$

Observing that $X(t)$ is an entire function, we obtain the series representation

$$
X(t)=\sum_{n=0}^{\infty} b_{n} t^{2 n}
$$

This series has the known zeros $t=(2 k \pi / \log 2) \pm i / 2$, where $k=0, \pm 1, \pm 2, \cdots$, together with an unlimited number of real zeros. So far neither the series (10) nor any of the chain of integrals leading up to it has yielded any new information relative to the verification of the hypothesis of Riemann about the nature of the zeros of $\zeta(s)$. If we get rid of the zeros of $X(t)$ corresponding to those of $\left(1-2^{s}\right)\left(1-2^{1-s}\right)$ by employing theta relations such as $2 \vartheta_{01}(2 x)=\vartheta_{00}(x)-\vartheta_{10}(x)$, then (7), or any of its consequences, reduces immediately to the corresponding result in the Riemann development.

CORNELL UNIVERSITY 